\title{
THE
}

UNIVERSITY

University of Rhode Island

OF RHODE ISLAND

DigitalCommons@URI

\section{On the linear approximation of mixture internal energies of departure}

\author{
Robert B. Kelly \\ University of Rhode Island \\ Angelo Lucia \\ University of Rhode Island, alucia@uri.edu
}

Follow this and additional works at: https://digitalcommons.uri.edu/che_facpubs

Part of the Chemical Engineering Commons

Creative Commons License

(c) (i) (9)

This work is licensed under a Creative Commons Attribution-Noncommercial-No Derivative Works 4.0 License.

\section{Citation/Publisher Attribution}

Kelly, R. B., \& Lucia, A. (2016). On the linear approximation of mixture internal energies of departure. Computers \& Chemical Engineering, 85. 72-75. doi: 10.1016/j.compchemeng.2015.10.013 Available at: https://doi.org/10.1016/j.compchemeng.2015.10.013

This Article is brought to you for free and open access by the Chemical Engineering at DigitalCommons@URI. It has been accepted for inclusion in Chemical Engineering Faculty Publications by an authorized administrator of DigitalCommons@URI. For more information, please contact digitalcommons-group@uri.edu. 
\& Chemical Engineering

Manuscript Number: CACE-D-15-00583R1

Title: On the Linear Approximation of Mixture Internal Energies of Departure

Article Type: Short Note

Section/Category: Modeling, numerical analysis and simulation

Keywords: internal energy of departure

direct Monte Carlo simulation

linear mixing rule

geological binary mixtures

equations of state

Corresponding Author: Dr. Angelo Lucia,

Corresponding Author's Institution: University of Rhode Island

First Author: Angelo Lucia

Order of Authors: Angelo Lucia; Robert B Kelly 
Professor Rafiq Gani, Editor

Computers \& Chemical Engineering Journal

Dear Professor Gani:

Please consider the revised article entitled " On the Linear Approximation of Mixture Internal Energies of Departure" by R.B. Kelly and A. Lucia for publication as a Short Note in Computers and Chemical Engineering.

Sincerely,

Angelo Lucia 


\section{Detailed Response to Reviews}

In the revision, we have addressed all reviewer comments regarding the use of Monte Carlo simulation section 2.4 and the sensitivity of the mixture energy parameter to uncertainty in the internal energy of departure in section 4 - both of which are additions to the original manuscript. More specifically we have

1) Added a flowchart description of the Monte Carlo simulation methods used in our work in section 2.3.

2) Validated the use of Monte Carlo simulation by comparisons with simulations of other workers in the field and experimental data in Section 2.4 of the revised manuscript.

3) Added an analysis of the sensitivity of the mixture energy parameter and mixture molar volume to uncertainty in internal energies of departure and provided two illustrations in Figures 2 and 3. 


\section{Highlights}

- Linear mixing rule for mixture internal energies of departure validated

- Comparisons are made to direct Monte Carlo simulation in the NPT ensemble

- Gas-oil, oil-oil and oil-water mixtures are considered

- Sensitivity analysis of $a_{M}$ and $V_{M}$ to uncertainty in $U_{M}^{D}$ presented 


\title{
On the Linear Approximation of Mixture Internal Energies of Departure
}

\author{
Robert B. Kelly and Angelo Lucia* \\ Department of Chemical Engineering, University of Rhode Island, Kingston, RI, 02881, United States \\ *Corresponding author. Tel.: +1 4018742814. \\ E-mail address: alucia@uri.edu (A. Lucia).
}

\begin{abstract}
Direct Monte Carlo simulation of internal energies of departure for binary mixtures of geological interest are gathered and compared to those calculated using a linear mixing rule. Simulation results for gas-oil, oil-oil, and oil-water mixtures show that the linear mixing rule used in the Gibbs-Helmholtz Constrained (GHC) equation of state framework gives accurate approximations of binary mixture internal energies of departure. A flowchart for computing internal energies of departure using Monte Carlo simulation is included along with a sensitivity analysis for the GHC mixture energy parameter with respect to uncertainty in internal energies of departure.
\end{abstract}

Keywords: internal energy of departure; direct Monte Carlo simulation; linear mixing rule; geological binary mixtures; equations of state 


\section{Introduction}

The Gibbs-Helmholtz Constrained (GHC) equation of state (EOS) is a multi-scale adaptation of the Soave form (Soave 1972) of the Redlich-Kwong EOS (Redlich and Kwong 1949),

$P=\frac{R T}{V-b}-\frac{a}{V(V+b)}$

where the energy parameter, $a$, given by

$a(T, P)=\left[\frac{0.42748 R^{2} T_{c}}{P_{c}}+\frac{b U^{D}}{T_{c} \ln 2}+\frac{2 b R \ln T_{c}}{\ln 2}\right] T-\frac{b U^{D}}{\ln 2}-\left[\frac{2 b R}{\ln 2}\right] T \ln T$

is derived analytically by constraining $a(T, P)$ to satisfy the Gibbs-Helmholtz equation

$$
\left(\frac{\partial \ln \varphi}{\partial T}\right)_{P}=-\frac{H^{D}}{R T^{2}}
$$

The resulting expression for the energy parameter shown in Eq. 2 is an explicit function of temperature and has implicit dependence on temperature and pressure through the functionality of the internal energy of departure, $U^{D}$. The dependence of the energy parameter on $U^{D}$ - which can be computed for pure components and mixtures via molecular simulation - makes the GHC equation a multi-scale EOS.

For mixtures, single fluid theory is applied to the EOS so that Kay's rules

$M_{c M}=\sum_{i=1}^{C} x_{i} M_{c i}$

are used for estimating mixture critical properties ( $M$ is any critical property) and linear mixing rules

$b_{M}=\sum_{i=1}^{C} x_{i} b_{i}$

$U_{M}^{D}=\sum_{i=1}^{C} x_{i} U_{i}^{D}$

are used for the mixture molecular co-volume, $b_{M}$, and mixture internal energy of departure, $U_{M}^{D}$, respectively. These quantities are needed in the expression for the mixture energy parameter, which is given by 
$a_{M}(T, P, x)=\left[\frac{0.42748 R^{2} T_{c M}}{P_{c M}}+\frac{b_{M} U_{M}^{D}}{T_{c M} \ln 2}+\frac{2 b_{M} R \ln T_{c M}}{\ln 2}\right] T-\frac{b_{M} U_{M}^{D}}{\ln 2}-\left[\frac{2 b_{M} R}{\ln 2}\right] T \ln T$

Furthermore, the GHC equation, originally proposed by Lucia (2010), is thermodynamically consistent (Lucia and Henley 2013) and has been used successfully to predict density and phase equilibrium of geological systems such as carbon dioxide-aqueous electrolytes mixtures (Lucia et al., 2012), hexagonal ice and structure I hydrates (Henley et al., 2014), and salt precipitation in systems with multiple salts (Lucia et al., 2015).

The objective of this note is to present numerical evidence that shows that the linear mixing for $U_{M}^{D}$ is valid for gas-oil, oil-oil, and oil-water mixtures. Accordingly, the sections of this short note are organized as follows. Section 2 provides molecular simulation details, describes the computational procedure used to determine binary mixture $U_{M}^{D}$, and validates the use of Monte Carlo simulation. Section 3 compares results for $U_{M}^{D}$ from direct Monte Carlo simulation with those using the linear mixing rule. Section 4 gives a sensitivity analysis of the GHC mixture energy parameter with respect to uncertainty in $U_{M}^{D}$ and provides two numerical illustrations. Finally, closing remarks are given in Section 5.

\section{Computational Procedure for the Internal Energy of Departure}

This section presents many of the details of the Monte Carlo simulations in this work.

\subsection{Internal Energy of Departure}

The internal energy of departure is defined as

$U^{D}(T, P)=U(T, P)-U^{i g}(T)$

where the superscript ig denotes ideal gas. 


\subsection{Simulation and Force Field Details}

MCCCS Towhee version 7.1.0 (Martin 2013) was used for all Monte Carlo simulations.

TraPPE-UA (Martin and Siepmann 1998; 1999) and TraPPE-EH (Potoff and Siepmann

2001) force field models were used for oil-oil and gas-oil mixtures using the parameters provided in Towhee. The TIP4P-Ew force field (Horn et al. 2004) was used for water and standard Lorentz-Berthelot mixing and combining rules were used for all mixtures.

\subsection{Molecular Simulation Procedure}

A flowchart for computing $\left\langle U_{i}^{D}\right\rangle$ and $\left\langle U_{M}^{D}\right\rangle$ is given in Figure 1 .

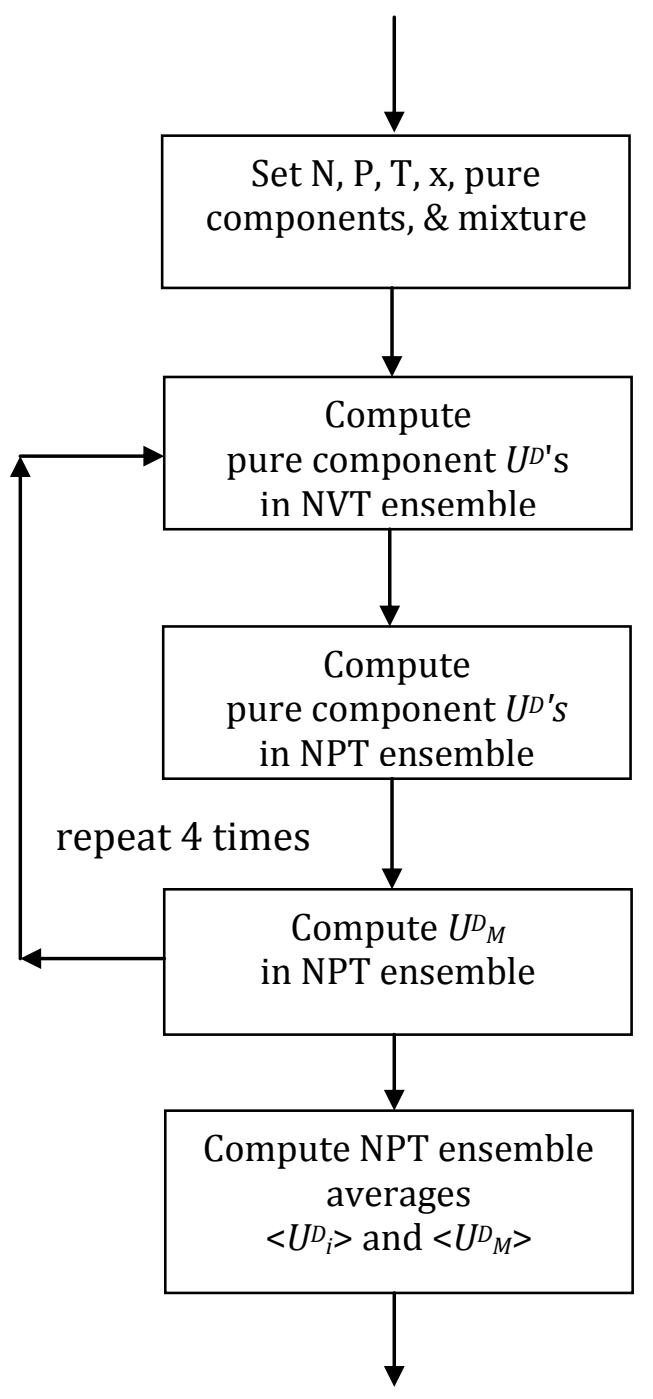

Figure 1: Flowchart for Computing Internal Energies of Departure 
For all ensembles, 40,000 - 100,000 equilibration and production cycles were used. The number of molecules was $\mathrm{N}=32-128$ for pure components (see Table 3 ) and $\mathrm{N}=100$ for mixtures. Mixture internal energies of departure were calculated for various compositions using the largest pure component radial cutoff distance(s). In the canonical (NVT) ensemble, reference state $U_{i}^{D}$ for each pure component account for intra-molecular interactions (i.e. electrostatic, torsion, etc.) and were computed by placing a single molecule in a box with radial cutoff distance(s) large enough to capture all intra-molecular effects. Ensemble averages, $\left\langle U^{D_{i}}>\right.$ and $\left\langle U_{M}^{D}>\right.$, were calculated by set averaging.

\subsection{Are Monte Carlo Simulation Results Accurate?}

Comparisons of calculated properties with experimental data are generally used to validate the accuracy of Monte Carlo simulation results. For example, Table 4 in Martin and Siepmann (1998) shows a comparison of experimental saturated liquid densities at standard conditions with those computed using the TraPPE force field for n-pentane, $n$ octane, and n-dodecane. Average root mean square errors reported in that table are quite small (i.e., $\sim 10^{-3}$ ). Table 1 shows that the Monte Carlo simulation densities in this work match both those from Martin and Siepmann (1998) and experimental data quite well.

Horn et al. (Table V, p. 9672, 2004) have validated the TIP4P-Ew model with experimental data for liquid water for a wide range of conditions and have calculated average densities and heats of vaporization, which show very good agreement with experimental data. In addition, they report average $U^{D}$ values for water over a range of temperatures, which allows direct comparison with results in this work. Specifically, Table 2 shows that the 
TIP4P-Ew simulation results for $<U^{D}>$ of water used in this work closely matches those reported in Horn et al., where slight differences can be attributed to the statistical nature of Monte Carlo simulation and ensemble size.

In our opinion, these comparisons demonstrate that the Monte Carlo simulation results in this study represent accurate values of internal energies of departure.

\section{Main Computational Results and Discussion}

Table 3 lists pure component data used in this study while Tables 4-6 show comparisons of $<U_{M}^{D}>$ from direct Monte Carlo simulation of mixtures with those for the linear mixing rule for n-octane-methane, 5-butylnonane-carbon dioxide, and n-heptane-n-dodecane. The numbers to the immediate right of $<U_{M}^{D}>$ shown in parentheses correspond to standard deviations. The average absolute deviations (AAD) \% errors for the linear mixing rule were $0.80,1.75$, and $0.73 \%$ respectively.

Table 7 lists values of $\left\langle U_{M}^{D}>\right.$ calculated from direct Monte Carlo simulation and the linear mixing rule for various compositions of n-hexane-water at $290 \mathrm{~K}$ and $150 \mathrm{bar}$. This mixture exhibits a rather large region of immiscibility (i.e., overall compositions ranging from $4.21 \times 10^{-10}$ to $99.996 \mathrm{~mol} \%$ hexane). Moreover, while the error is much larger in the region of immiscibility, this is unimportant in practice because the error in the linear mixing rule is small for dilute solutions of n-hexane and water, especially those far from the plait point. For example, Table 7 shows that for a mixture of $99 \mathrm{~mol} \% \mathrm{n}$-hexane and $1 \mathrm{~mol} \%$ water at $290 \mathrm{~K}$ and $150 \mathrm{bar}$, the linear mixing rule gives a value of $U_{M}^{D}=-281959 \mathrm{~cm}^{3} \mathrm{bar} / \mathrm{mol}$, which is an error of $1.99 \%$. A small error is also observed for the water-rich composition of 99 mol\% water-1 mol\% n-hexane. 


\section{Sensitivity Analysis}

Figure 7 in Lucia et al. (p. 85, 2012) shows that $U^{D}$ has a small impact on the molar volume, $V_{M}$, of liquid water. In this section, a more detailed study of the sensitivity of $a_{M}$ and $V_{M}$ with respect to $U_{M}^{D}$ is presented. From Eq. 7, it is easily seen that the change in $a_{M}$ with respect to changes in $U_{M}^{D}$ is given by

$\left(\frac{\partial a_{M}}{\partial U_{M}^{D}}\right)=\left[\frac{T}{T_{c M}}-1\right]\left(\frac{b_{M}}{\ln 2}\right)$

and the local change in $a_{M}$ with respect to changes (or uncertainty) in $U_{M}^{D}$ is given by

$\Delta a_{M}=\left[\frac{T}{T_{c M}}-1\right]\left(\frac{b_{M}}{\ln 2}\right) \Delta U_{M}^{D}=\left[\frac{T}{T_{c M}}-1\right]\left(\frac{b_{M}}{\ln 2}\right) \sum_{i=1}^{C} x_{i} \Delta U_{i}^{D}$

where $\Delta$ denotes perturbation. Figure 2 gives plots showing relative changes in $a_{M}$ for $\Delta U_{i}^{D}=0.05 U_{i}^{D}, i=1,2$ as a function of composition for two mixtures studied in Section 3. Note that the relative sensitivity of $a_{M}$ to $5 \%$ change in $U_{M}^{D}$ is $<4 \%$ everywhere and for methane-octane it is zero at $x_{C H 4} \approx 0.7$. This is because $a_{M}$ is independent of $U_{M}^{D}$ at the point where $T_{c M}$ from Kay's rule is equal to the specified temperature $T=300 \mathrm{~K}$.

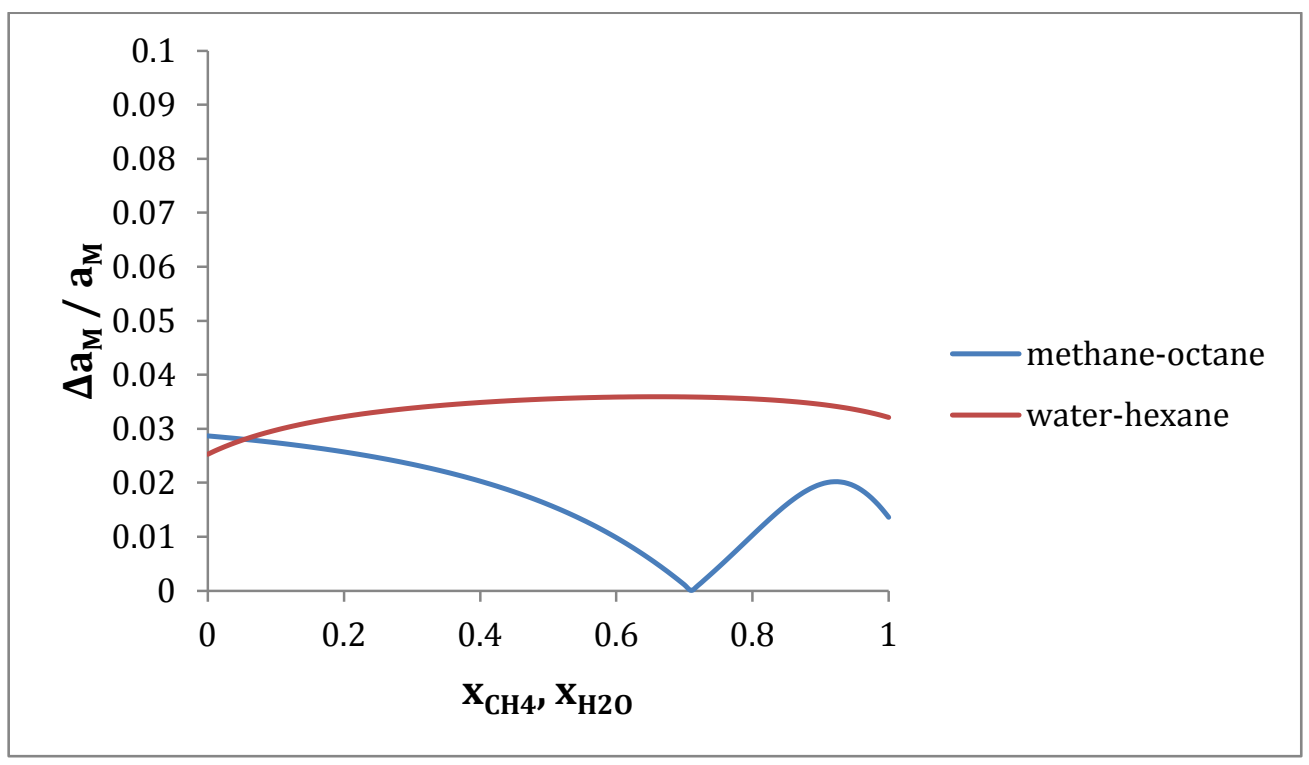

Figure 2: Sensitivity of $\boldsymbol{a}_{\boldsymbol{M}}$ to $\mathbf{5 \%}$ Change in $U_{M}^{D}$ 
Figure 3 shows the corresponding relative sensitivity of molar volume, where molar volumes were computed by the GHC EOS using the pure component data shown in Table 8 .

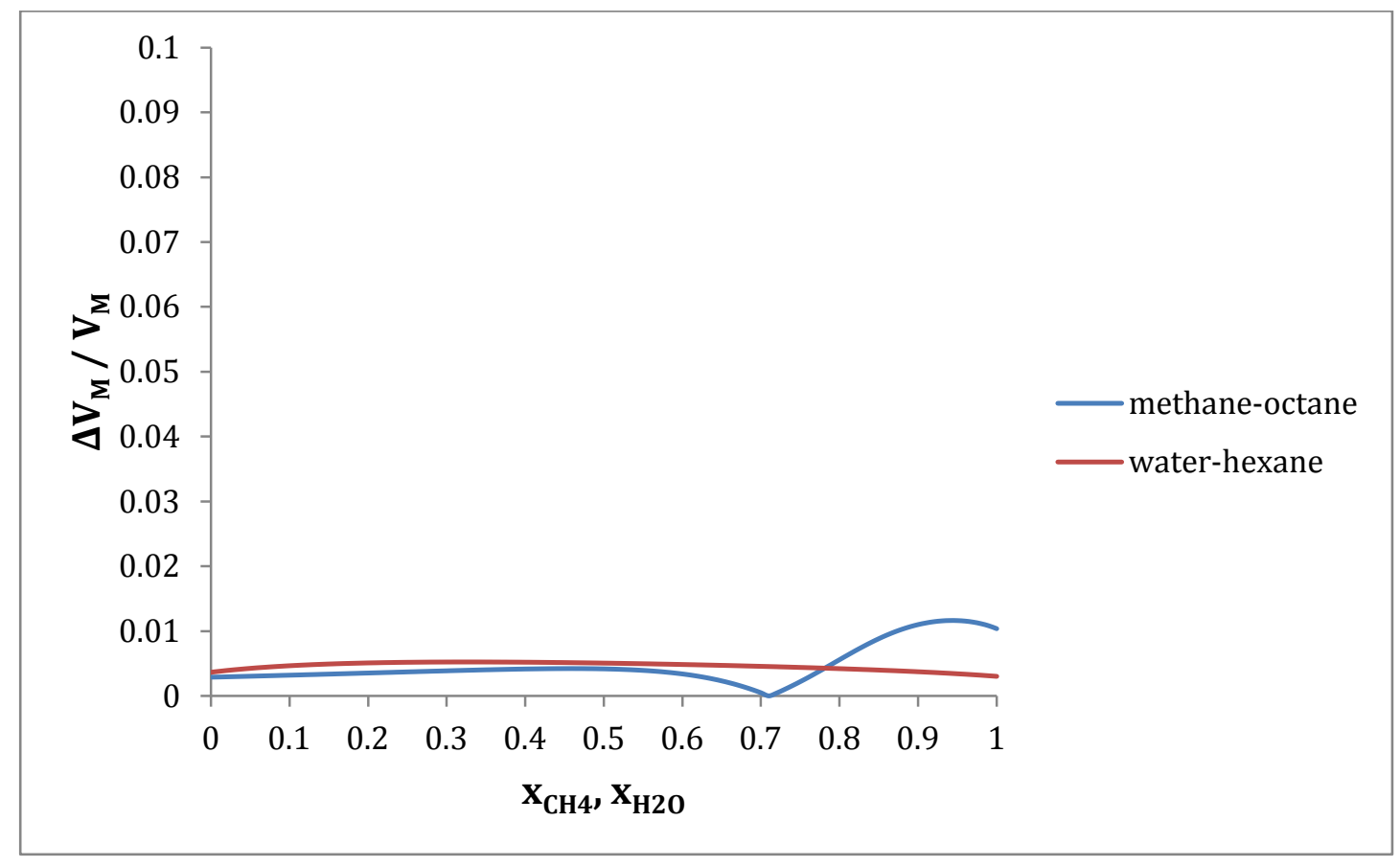

Figure 3: Sensitivity of $V_{M}$ to $5 \%$ Change in $U_{M}^{D}$

Note that the relative sensitivity of $V_{M}$ to changes in $U_{M}^{D}$ is less than $1.5 \%$ over the entire composition range.

\section{Conclusions}

Direct Monte Carlo simulation was used to compare binary mixture internal energies of departure to those computed using the linear mixing proposed by Lucia et al. (2012). AAD\% errors clearly show that the linear mixing rule provides very good approximations of $U_{M}^{D}$ for gas-oil, oil-oil, and oil-water mixtures and validates the use of the linear mixing rule in the GHC EOS framework. Finally, a sensitivity analysis for the mixture energy parameter as a function of $U_{M}^{D}$ was conducted. Results show that uncertainty in $U_{M}^{D}$ (from the linear mixing rule) has a small impact on $a_{M}$ and fluid molar volume. 
Table 1: Comparison of Monte Carlo Simulation Densities

\begin{tabular}{|c|c|c|c|}
\hline Species & $\rho(\mathrm{g} / \mathrm{mL})^{*}$ & $\rho(\mathrm{g} / \mathrm{mL})^{* *}$ & $\rho^{\exp }(\mathrm{g} / \mathrm{mL})^{*}$ \\
\hline n-octane & 0.705 & 0.704 & 0.7025 \\
\hline n-dodecane & 0.754 & 0.756 & 0.7487 \\
\hline
\end{tabular}

* taken from Table 4 in Martin \& Siepmann (1998)

** this work

Table 2: Comparison of $U^{D}$ for Water Using the TIP4P-Ew Force Field

\begin{tabular}{|c|c|c|c|}
\hline$T(K)$ & $U^{\mathrm{D}}(\mathrm{kcal} / \mathrm{mol}) *$ & $U^{\mathrm{D}}(\mathrm{kcal} / \mathrm{mol}) * *$ & $\%$ Difference \\
\hline 298 & -5687.4 & -5697.2 & 0.17 \\
\hline 310.5 & -5596.8 & -5603.3 & 0.12 \\
\hline 323 & -5496.6 & -5510.9 & 0.26 \\
\hline 335.5 & -5401.2 & -5418.4 & 0.32 \\
\hline 348 & -5311.0 & -5326.0 & 0.28 \\
\hline 360.5 & -5219.3 & -5236.1 & 0.32 \\
\hline 373 & -5128.2 & -5146.6 & 0.36 \\
\hline 400 & -4939.7 & -4953.4 & 0.27 \\
\hline
\end{tabular}

* taken from Table $\mathrm{V}$ in Horn et al. (2004)

** this work; $\mathrm{N}=32, \mathrm{P}=1$ bar

Table 3: Force Field, N, T, P, Ensemble Average, and Reference Internal Energy

\begin{tabular}{|c|c|c|c|c|c|c|}
\hline Species & Force Field & $\mathrm{N}$ & $\mathrm{T}(\mathrm{K})$ & $\mathrm{P}($ bar $)$ & $\left\langle U_{i}^{D}(T, P)\right\rangle$ & $\left\langle U_{i}^{o}(T, V)\right\rangle$ \\
\hline Methane & TraPPE-UA & 64 & 300 & 200 & $-23652(173)$ & -- \\
\hline Octane & TraPPE-UA & 64 & 300 & 200 & $-192344(221)$ & $177400(483)$ \\
\hline Carbon Dioxide & TraPPE-EH & 128 & 273.15 & 100 & $-112930(671)$ & -- \\
\hline 5-Butylnonane & TraPPE-UA & 64 & 273.15 & 100 & $-350234(2416)$ & $240875(568)$ \\
\hline Water & TIP4P-EW & 128 & 290 & 150 & $-470000(299)$ & -- \\
\hline Hexane & TraPPE-UA & 32 & 290 & 150 & $-170109(640)$ & $109950(58)$ \\
\hline Heptane & TraPPE-UA & 32 & 350 & 300 & $-132594(436)$ & $171700(163)$ \\
\hline Dodecane & TraPPE-UA & 32 & 350 & 300 & $-159070(1521)$ & $359150(640)$ \\
\hline
\end{tabular}

* All energies are in units of $\mathrm{cm}^{3} \mathrm{bar} / \mathrm{mol}$. 
Table 4: Comparison for Octane-Methane*

\begin{tabular}{|c|c|c|c|c|}
\hline $\mathrm{x}_{\text {octane }}$ & $\mathrm{x}_{\text {methane }}$ & $U_{M}^{D}(T, P)$ & $\sum_{i=1}^{C} x_{i} U_{i}^{D}(T, P)$ & $\%$ Error \\
\hline 0.80 & 0.20 & -297270 & -300525 & 1.10 \\
\hline 0.50 & 0.50 & -195125 & -196698 & 0.81 \\
\hline 0.30 & 0.70 & -126840 & -127480 & 0.50 \\
\cline { 3 - 4 } & \multicolumn{2}{|r}{} & AAD & 0.80 \\
\hline
\end{tabular}

$* N=100, T=300 \mathrm{~K}, \mathrm{P}=200$ bar. All energy units are in $\mathrm{cm}^{3} \mathrm{bar} / \mathrm{mol}$.

Table 5: Comparison for 5-Butylnonane-Carbon Dioxide*

\begin{tabular}{|c|c|c|c|c|}
\hline$X_{5 \text {-butylnonane }}$ & $\mathrm{X}_{\text {carbon dioxide }}$ & $U_{M}^{D}(T, P)$ & $\sum_{i=1}^{C} x_{i} U_{i}^{D}(T, P)$ & $\%$ Error \\
\hline 0.80 & 0.20 & -491200 & -495473 & 0.87 \\
\hline 0.50 & 0.50 & -343638 & -352020 & 2.44 \\
\hline 0.20 & 0.80 & -204600 & -208566 & 1.94 \\
\hline & & & AAD & 1.75 \\
\hline
\end{tabular}

* $N=100, T=273.15 \mathrm{~K}, \mathrm{P}=100 \mathrm{bar}$. All energy units are in $\mathrm{cm}^{3} \mathrm{bar} / \mathrm{mol}$.

Table 6: Comparison for Heptane-Dodecane*

\begin{tabular}{|c|c|c|c|c|}
\hline$x_{\text {heptane }}$ & $x_{\text {dodecane }}$ & $U_{M}^{D}(T, P)$ & $\sum_{i=1}^{C} x_{i} U_{i}^{D}(T, P)$ & $\%$ Error \\
\hline 0.75 & 0.25 & -353588 & -357775 & 1.18 \\
\hline 0.50 & 0.50 & -408900 & -411257 & 0.58 \\
\hline 0.25 & 0.75 & -462688 & -464739 & 0.44 \\
\hline \multicolumn{2}{r}{} & AAD & 0.73 \\
\hline
\end{tabular}

* $N=100, T=350 \mathrm{~K}, \mathrm{P}=300$ bar. All energy units are in $\mathrm{cm}^{3} \mathrm{bar} / \mathrm{mol}$.

Table 7: Comparison for Hexane-Water*

\begin{tabular}{|c|c|c|c|c|c|}
\hline $\mathrm{x}_{\text {water }}$ & $\mathrm{x}_{\text {hexane }}$ & $\begin{array}{c}\text { Single Phase } \\
\text { Stability }\end{array}$ & $U_{M}^{D}(T, P)$ & $\sum_{i=1}^{C} x_{i} U_{i}^{D}(T, P)$ & $\%$ Error \\
\hline 0.99 & 0.01 & Unstable & -467050 & -468101 & 0.23 \\
\hline 0.75 & 0.25 & Unstable & -386463 & -422515 & 9.33 \\
\hline 0.50 & 0.50 & Unstable & -338725 & -375030 & 10.72 \\
\hline 0.25 & 0.75 & Unstable & -301038 & -327545 & 8.81 \\
\hline 0.01 & 0.99 & Unstable & -276451 & -281959 & 1.99 \\
\cline { 5 - 5 }
\end{tabular}

${ }^{*} \mathrm{~N}=100, \mathrm{~T}=290 \mathrm{~K}, \mathrm{P}=150 \mathrm{bar}$. All energy units are in $\mathrm{cm}^{3} \mathrm{bar} / \mathrm{mol}$. 
Table 8: Pure Component Fluid Properties

\begin{tabular}{|c|c|c|c|}
\hline Species & $\mathrm{T}_{\mathrm{c}}(\mathrm{K})$ & $\mathrm{P}_{\mathrm{c}}(\mathrm{bar})$ & $\mathrm{b}\left(\mathrm{cm}^{3} / \mathrm{mol}\right)$ \\
\hline Methane & 190.58 & 45.92 & 29.614 \\
\hline Octane & 568.83 & 24.86 & 143.145 \\
\hline Water & 647.37 & 221.20 & 16.363 \\
\hline Hexane & 507.60 & 30.20 & 110.309 \\
\hline
\end{tabular}

\begin{tabular}{|ll|}
\hline Nomenclature & \\
$a, a_{M}$ & pure component liquid energy parameter, liquid mixture energy parameter \\
$b, b_{i}, b_{M}$ & molecular co-volume, ith component molecular co-volume, mixture molecular \\
& co-volume \\
$C$ & number of components \\
$H^{D}$ & enthalpy of departure \\
$\mathrm{N}$ & number of molecules \\
$P, P_{c}, P_{c M}$ & pressure, critical pressure, mixture critical pressure \\
$R$ & gas constant \\
$T, T_{c}, T_{c M}$ & absolute temperature, critical temperature, mixture critical temperature \\
$U_{i}^{D}, U_{i}^{o}$ & $\mathrm{i}^{\text {th }}$ component internal energy of departure, reference state internal energy \\
$V_{M}$ & mixture molar volume \\
$x, x_{i}$ & mole fraction, ith component mole fraction \\
& \\
$G r e e k$ Symbols & fugacity coefficient \\
$\varphi$ & mass density \\
$\rho$ &
\end{tabular}

\section{References}

Henley, H., Thomas, E., \& Lucia, A. (2014). Density and phase equilibrium for ice and structure I hydrates using the Gibbs-Helmholtz constrained equation of state. Chemical Engineering Research and Design, 92(12), 1977-1991. doi:10.1016/j.cherd.2014.06.011

Horn, H. W., Swope, W. C., Pitera, J. W., Madura, J. D., Dick, T. J., Hura, G. L., \& Head-Gordon, T. (2004). Development of an improved four-site water model for biomolecular simulations: TIP4P-Ew. Journal of Chemical Physics, 120(20), 9665-9678. doi:10.1063/1.1683075

Lucia, A. (2010). A multi-scale Gibbs-Helmholtz Constrained cubic equation of state. Journal of Thermodynamics, 2010, 1-10. doi:10.1155/2010/238365 
Lucia, A., Bonk, B. M., Waterman, R. R., \& Roy, A. (2012). A multi-scale framework for multiphase equilibrium flash. Computers and Chemical Engineering, 36, 79-98. doi:10.1016/j.compchemeng.2011.07.011

Lucia, A., \& Henley, H. (2013). Thermodynamic consistency of the multi-scale Gibbs Helmholtz constrained equation of state. Chemical Engineering Research and Design, 91, 1748-1759. doi:10.1016/j.cherd.2013.03.009

Lucia, A., Henley, H., \& Thomas, E. (2014). Multiphase equilibrium flash with salt precipitation in systems with multiple salts. Chemical Engineering Research and Design, 93, 662-674. doi:10.1016/j.cherd.2014.04.034

Martin, M. G., \& Siepmann, J. I. (1998). Transferable potentials for phase equilibria. 1. United-atom description of $\mathrm{n}$-alkanes. The Journal of Physical Chemistry B, 102(97), 2569-2577. doi:10.1021/jp972543+

Martin, M. G., \& Siepmann, J. I. (1999). Novel configurational-bias Monte Carlo method for branched molecules. Transferable potentials for phase equilibria. 2. United-atom description of branched alkanes. The Journal of Physical Chemistry B, 103(21), 45084517. doi:10.1021/jp984742e

Martin, M. G. (2013). MCCCS Towhee: a tool for Monte Carlo molecular simulation. Molecular Simulation, 39(14-15), 1212-1222. doi:10.1080/08927022.2013.828208

Potoff, J. J., \& Siepmann, J. I. (2001). Vapor-liquid equilibria of mixtures containing alkanes, carbon dioxide, and nitrogen. AIChE Journal, 47(7), 1676-1682.

doi:10.1002/aic.690470719

Redlich, O., \& Kwong, J. N. S. (1949). On the thermodynamics of solutions; an equation of state; fugacities of gaseous solutions. Chemical Reviews, 44(1), 233-244.

doi:10.1021/cr60137a013

Soave, G. (1972). Equilibrium constants from a modified Redlich-Kwong equation of state. Chemical Engineering Science, 27(6), 1197-1203. doi:10.1016/0009-2509(72) 